\title{
Coherent transport structures in magnetized plasmas. I. Theory
}

G. Di Giannatale, M. V. Falessi, D. Grasso, F. Pegoraro, and T. J. Schep

Citation: Physics of Plasmas 25, 052306 (2018); doi: 10.1063/1.5020163

View online: https://doi.org/10.1063/1.5020163

View Table of Contents: http://aip.scitation.org/toc/php/25/5

Published by the American Institute of Physics

\section{Articles you may be interested in}

Coherent transport structures in magnetized plasmas. II. Numerical results

Physics of Plasmas 25, 052307 (2018); 10.1063/1.5020164

Fluid simulations of plasma turbulence at ion scales: Comparison with Vlasov-Maxwell simulations

Physics of Plasmas 25, 052302 (2018); 10.1063/1.5026656

Three-dimensional particle-particle simulations: Dependence of relaxation time on plasma parameter

Physics of Plasmas 25, 052112 (2018); 10.1063/1.5025431

$\mathrm{E} \times \mathrm{B}$ electron drift instability in Hall thrusters: Particle-in-cell simulations vs. theory

Physics of Plasmas 25, 061204 (2018); 10.1063/1.5017033

Gyrokinetic theory of slab universal modes and the non-existence of the gradient drift coupling (GDC) instability Physics of Plasmas 25, 052115 (2018); 10.1063/1.5024748

Energy spectrum of tearing mode turbulence in sheared background field

Physics of Plasmas 25, 062305 (2018); 10.1063/1.5022292

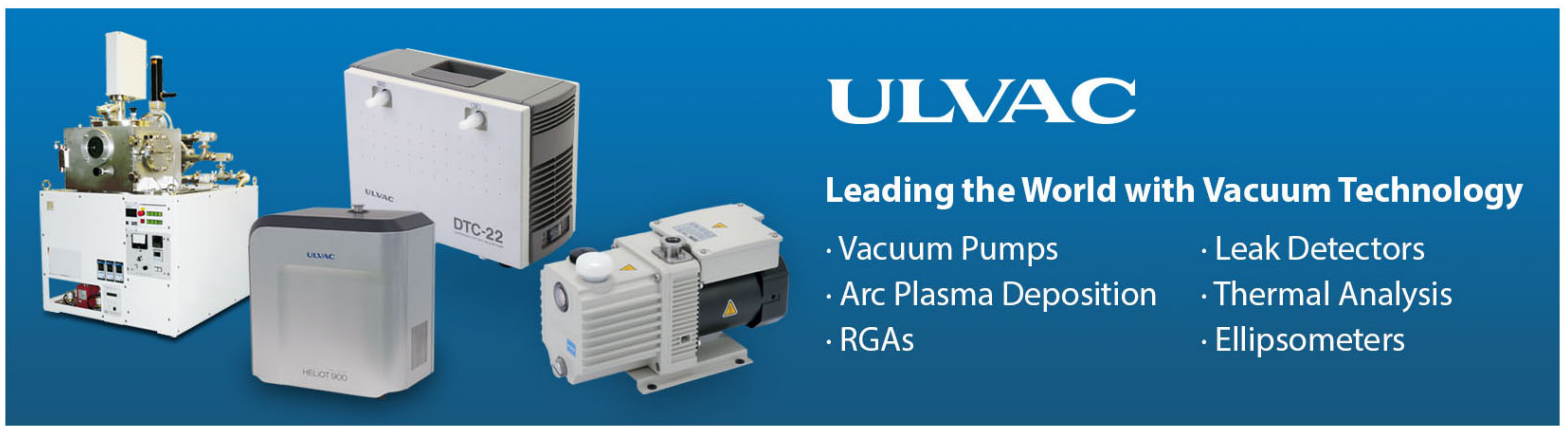




\title{
Coherent transport structures in magnetized plasmas. I. Theory
}

\author{
G. Di Giannatale, ${ }^{1}$ M. V. Falessi, ${ }^{2}$ D. Grasso, ${ }^{3}$ F. Pegoraro, ${ }^{4}$ and T. J. Schep ${ }^{5}$ \\ ${ }^{1}$ IGI-CNR Corso Stati Uniti 4, Padova, Italy \\ ${ }^{2}$ Dipartimento di Matematica e Fisica, Roma Tre University, Roma, Italy \\ ${ }^{3}$ ISC - CNR and Politecnico di Torino Dipartimento Energia, C.so Duca degli Abruzzi 24, Torino, Italy \\ ${ }^{4}$ Dipartimento di Fisica E. Fermi, Pisa University, largo Pontecorvo 3, Pisa, Italy \\ ${ }^{5}$ Fluid Dynamics Laboratory, Department of Applied Physics, Eindhoven University of Technology, \\ P. O. Box 513, 5600MB Eindhoven, The Netherlands
}

(Received 20 December 2017; accepted 23 April 2018; published online 25 May 2018)

In a pair of linked articles (called Papers I and II, respectively), we apply the concept of Lagrangian Coherent Structures (LCSs) borrowed from the study of dynamical systems to magnetic field configurations in order to separate regions where field lines have a different kind of behaviour. In the present article, Paper I, after recalling the definition and the properties of the LCSs, we show how this conceptual framework can be applied to the study of particle transport in a magnetized plasma. Furthermore, we introduce a simplified model that allows us to consider explicitly the case where the magnetic configuration evolves in time on time scales comparable to the particle transit time through the configuration. In contrast with previous works on this topic, this analysis requires that a system that is aperiodic in time be investigated. Published by AIP Publishing.

https://doi.org/10.1063/1.5020163

\section{INTRODUCTION}

The understanding of transport phenomena in magnetized plasmas is notoriously one of the most challenging tasks in the investigation of both laboratory and space plasmas. This is particularly the case in low collisionality plasmas where particles are essentially free to stream along magnetic field lines while their transport in the perpendicular direction is governed by collective electric and magnetic fluctuations and not by binary interactions. As a consequence, transport is generally anisotropic, nonlocal and, most likely, not described by simple diffusion equations.

In recent years, the concept of Lagrangian Coherent Structures (LCSs) has been introduced by Haller in the context of transport processes in complex fluid flows, see Ref. 1. In a two-dimensional configuration, these structures are now defined as special lines which are advected by the fluid and which organize the flow, e.g., see Refs. 2 and 3. The importance of the LCS stems from the fact that they are a generalization of the dynamical structures observed in autonomous and periodic systems, e.g., invariant manifolds, to temporally aperiodic flows, see, e.g., Ref. 2. Analogously to these structures, they separate the flow domain into macro-regions inside which fast mixing phenomena take place. Over the finite time span which characterizes the LCSs, these macro-regions do not exchange fluid elements and thus act as transport barriers. The LCS concept provides a very effective tool that is being increasingly used in order to describe transport processes in a wide range of conditions: the pollutant transport on the ocean surface, ${ }^{4}$ blood flow, ${ }^{5}$ the spreading of plankton blooms, ${ }^{6}$ jelly fish predator-prey interaction, ${ }^{7}$ atmospheric dataset analysis, ${ }^{8}$ transport features of the beam-plasma instability, ${ }^{9}$ solar photospheric flows, ${ }^{10}$ saturation of a nonlinear dynamo, ${ }^{11}$ and in magnetized plasmas. ${ }^{12-14}$ A different kind of LCS, called invariant-torus-like LCS, has been used in Ref. 15 for
Hamiltonian systems in which the perturbations vanish at some defined positions. In this context, we recall that other types of indicators, such as orbit stickiness, finite time rotation number, and braiding exponents, have also been used in the literature in order to delineate regions with different dynamical properties in Hamiltonian systems. ${ }^{12,13,16-18}$

Since their introduction, different mathematical definitions of LCS have been proposed with the aim of providing a tool capable of giving a concise representation of the transport processes. The earlier definition involved second derivative ridges of the finite time Lyapunov exponent field, e.g., see Ref. 19, but was later corrected and reformulated in terms of most repulsive or attractive material lines (see in Sec. V A). In addition, numerical procedures have been devised in order to extract such structures from fluid simulation results or, even more interestingly, from actual experimental data (Refs. 20 and 21).

In a number of recent articles, Refs. 22-24, the description of transport phenomena in magnetized plasmas has been addressed using LCS as a tool aimed at identifying transport barriers in a toroidal magnetic configuration in the presence of magnetic reconnection events. In a very recent article, see Ref. 25, the LCS tool has been used to show how applying boundary magnetic perturbations with different helicities gives rise to different transport barriers.

Both the second derivative ridges and the most repulsive or attractive material lines definitions have been adopted in Ref. 22 and in Ref. 24, respectively, and results obtained with the two different approaches have been compared in Ref. 24.

In these articles, the structure of the magnetic field lines has been used as a proxy for the structure of the particle trajectories, assuming that particles move along magnetic field lines as obtained from a "snapshot" of the magnetic configuration taken at a fixed time, neglecting finite particle orbit 
size effects, secular drifts, and assuming that the magnetic configuration does not evolve significantly on the particle transit time through the configuration.

In the present paper, the first one of a linked pair (called Papers I and II, respectively), after briefly revisiting the "snapshot" results, we formulate a generalization of the method that, while still not addressing the full particle dynamics, takes nevertheless into account the fact that the magnetic configuration may evolve on time scales comparable to the particle transit time. A consequence of this generalization is that the system becomes intrinsically aperiodic. We define velocity dependent LCS, i.e., introduce a rudimentary kinetic treatment that addresses the fact that particles with different energies can be expected to experience different transport barriers, as has been recently proved by means of test particles simulations in Ref. 26. After these definitions are established, we will use this generalization in the accompanying Paper II $^{62}$ in order to identify the LCSs in a magnetic configuration where magnetic reconnection evolves by referring to the same numerical simulation results that were used in Refs. 22 and 24. We remark that our choice of using the magnetic field lines as a proxy for the particle trajectories, along the lines, e.g., Ref. 27, is motivated by the aim to provide a tool that depends on as few physical parameters as is meaningful and that can be used in a general setting for an easy detection of barriers to the particle transport enhanced, as is the case considered here, by the growth of multiple island chains due to the onset of magnetic reconnection. A more quantitative justification of such a choice for the magnetic configuration studied here will be given in Sec. VI. More exact treatments that properly distinguish between particle trajectories and magnetic field lines and that involve the particle Hamiltonian, or the particle gyro-center Hamiltonian, are available (see, e.g., Refs. 28 and 29) but their application requires in a general setting a less straightforward procedure.

This paper is organized as follows. In Sec. II, the Hamiltonian nature of the magnetic field line equations is briefly rederived in order to illustrate the relationship with the dynamics of one-dimensional nonautonomous dynamical systems having in mind, as will be repeated later in the text, that the "time" entering in the Hamilton equations for the magnetic field lines is not the physical time but a properly chosen coordinate along field lines. A simplified planar magnetic configuration with a strong magnetic field component out of the plane (the so-called guide field) is considered. The fact that it corresponds to a non-autonomous dynamical system is related to the effect of a process of magnetic reconnection that has broken the underlying structure of magnetic surfaces. This latter configuration would have corresponded to an autonomous (and thus integrable) one dimensional system. Then the connection with particle transport is recalled and some related early references are mentioned.

In Sec. II, the distinction between time periodic and aperiodic dynamical systems is made in connection with the different mathematical tools that are best suited to describe their dynamics. The role of the Poincare map is recalled together with a short overview of the so called lobe dynamics. The Poincaré map approach makes it possible to partition the phase space of the time periodic dynamical system into macro-regions distinguished by a qualitatively different behaviour of the trajectories they contain, e.g., periodic or chaotic trajectories.

In Sec. IV, the concept of LCSs is introduced. We close this section by briefly contrasting the initial definitions of LCSs in terms of second derivative ridges, e.g., see Ref. 19, and the definition in terms of maximal repelling and maximal attracting material lines.

In Sec. VI, we first describe (Sec. VIA) the time periodic dynamical system related to the magnetic configuration that we choose (see also Refs. 22 and 30) for the study of particle transport in the presence of magnetic reconnection. Then in Sec. VI B, we introduce a time nonperiodic dynamical system obtained by including in a simplified way the effect of the change of the magnetic configuration during the particle transit through it. This is done by combining the coordinate-like "time" entering in the Hamilton equations for the magnetic field lines mentioned above with the physical time that describes the change of the magnetic configuration caused, in our case of interest, by the onset of magnetic reconnection. The resulting "effective" time depends on the velocity of the specific particle that is considered and can be thought as the physical time of change of the local value of the magnetic field seen by the particle along its trajectory because of the combined effect of the magnetic field spatial inhomogeneity and physical time evolution. The numerical investigation of both the time periodic and the non-periodic systems will be described in the accompanying paper, Paper II.

\section{MAGNETIC FIELD AS A DYNAMICAL SYSTEM}

As is well known, ${ }^{31-36}$ due to their solenoidal nature, the field lines of a magnetic field in three-dimensional space that does not vanish within the domain of interest can be described at any fixed physical time $t=\bar{t}$ as trajectories of a non-autonomous Hamiltonian system with one degree of freedom. The role of time is played by a spatial coordinate taken to label the points along a field line. A simple derivation in terms of a general set of (curvilinear) coordinates $\chi_{i}$, $i=1,2,3$ can be given by choosing a gauge condition for the vector potential A such that one of its components, e.g., $A_{3}$, vanishes, i.e.,

$$
\begin{aligned}
& \mathbf{A}=A_{1} \nabla \chi_{1}+A_{2} \nabla \chi_{2}, \quad \text { and } \\
& \mathbf{B}=\nabla A_{1} \times \nabla \chi_{1}+\nabla A_{2} \times \nabla \chi_{2} .
\end{aligned}
$$

Since $\mathbf{B} \neq 0$ within the considered spatial domain, we can set $\mathbf{B} \cdot \nabla \chi_{1} \neq 0$. It follows that the Jacobian $\left(\nabla A_{2} \times \nabla \chi_{2}\right)$ $\cdot \nabla \chi_{1} \neq 0$, i.e., that the coordinate transformation to the new set of spatial coordinates $A_{2}, \chi_{2}, \chi_{1}$ is invertible.

Using $\mathbf{B}=\nabla A_{1}\left(\chi_{1}, \chi_{2}, A_{2}\right) \times \nabla \chi_{1}+\nabla A_{2} \times \nabla \chi_{2}$, from the field line condition $d \mathbf{l} \times \mathbf{B}=0$, we obtain the Hamilton equations

$$
\begin{aligned}
& \frac{d A_{2}}{d \chi_{1}}=\frac{\partial A_{1}}{\partial \chi_{2}}, \quad \frac{d \chi_{2}}{d \chi_{1}}=-\frac{\partial A_{1}}{\partial A_{2}} \\
& \text { together with } \quad \frac{d A_{1}}{d \chi_{1}}=\frac{\partial A_{1}}{\partial \chi_{1}} .
\end{aligned}
$$


In the above equations, $\chi_{1}$ plays the role of the "time" variable, and $\chi_{2}$ and $A_{2}$ that of the two canonical coordinates: $d A_{2} \leftrightarrow d q$ and $d \chi_{2} \leftrightarrow d p$, and $A_{1}\left(\chi_{2}, A_{2}, \chi_{1}\right)$ of the "Hamiltonian" $\mathcal{H}$. We anticipate here that in the following we will consider a simple magnetic field configuration often used in the study of magnetically confined plasmas where $\mathbf{B}=B_{z} \mathbf{e}_{z}+\nabla \psi \times \mathbf{e}_{z}$, with $B_{z}$ spatially uniform and $\mathbf{e}_{z}=\nabla z$. It corresponds, after an appropriate rescaling, to $\chi_{1}=z, \chi_{2}=x$, $A_{2}=y$ and $A_{1}(x, y, z)$ the so-called "poloidal" flux function $\psi(x, y, z, t=\bar{t})$.

The importance of this Hamiltonian formulation stems from the fact that it establishes a direct connection between magnetic configurations and dynamical systems, see, e.g., Refs. 28 and 37-40, and thus makes it possible to describe the topology of the magnetic field lines in terms of that of the trajectories of a dynamical system. Furthermore, if we assume that in the considered magnetic configuration an adiabatic approximation holds for the motion of the charged plasma particles, in the limit where their Larmor radius is negligible and disregarding the particle drifts, we can approximate their motion as occurring along magnetic field lines. Such an approximation allows us to study the particle advection and diffusion by using the same set of equations that determine the magnetic field lines, see, e.g., Ref. 27. This approach requires in addition that, as a first approximation, the change of the magnetic configuration during the particle motion be neglected.

The equivalence between the magnetic field lines and the trajectories of non-autonomous Hamiltonian systems with one degree of freedom has been widely used in the literature by adopting the concepts that are proper of dynamical systems; see, in particular, Ref. 31 and more recently Refs. 41-43.

In the case of magnetically confined plasmas, this equivalence has been used, in particular, in order to assess the effects of magnetic field lines reconnection events on the particle transport. In the context of the present article, we will refer to Ref. 30 and more specifically to Refs. 22 and 24 where dynamical system tools are used in order to characterize transport processes associated with the magnetic field lines topology. This approach makes it possible to partition the magnetic configuration into sub-domains characterized by different transport phenomena and, in particular, to identify the domains where they are either fastest or slowest.

\section{TRANSPORT PHENOMENA IN NON- AUTONOMOUS, TIME PERIODIC, DYNAMICAL SYSTEMS WITH ONE DEGREE OF FREEDOM}

Non-autonomous dynamical systems may have a periodic or non-periodic time dependence, see, e.g., Refs. 44-46, and the techniques used to study these two cases may differ. In particular, the Poincaré section (stroboscopic map) method can be used to reduce the dimensionality of the problem by studying, see, e.g., Ref. 47 and references therein, a $2 N$ dimensional map instead of a $2 N+1$ continuous-time dynamical system. For a periodic system, this map is constructed by associating with an initial condition its evolution after one period. The main advantage obtained by using this technique is to filter out redundant dynamical phenomena and reveal the underlying nature of the motion, e.g., whether it is regular or chaotic. Furthermore, invariant curves of the Poincare map can be used to partition the phase space into regions where trajectories have a qualitatively different behaviour on a given time scale, e.g., bounded or unbounded. ${ }^{48-50}$ These structures play a fundamental role in governing transport processes in non-autonomous dynamical systems and, in particular, they determine the so-called lobe dynamics. Here we will briefly recall a few definitions, in particular, the definition of invariant manifolds that are needed in the following in connection with the LCSs, while extensive presentations can be found in Refs. 51-54. In view of the present application to the study of the topology of magnetic field lines at fixed physical time, we consider explicitly systems with one degree of freedom $(\mathrm{N}=1)$. Following Ref. 54, a lobe is defined as a region of the extended $(2 N+1$ dimensional) phase space enclosed by segments of the intersection between stable and unstable manifolds and a $t=$ const surface (i.e., here, at a $z=$ const surface, this remark will not be repeated in the rest of the section and in the following ones until the true physical time is reintroduced in Sec. VI B). Stable and unstable manifolds are defined with respect to a distinguished hyperbolic trajectory (DHT), i.e., with respect to a special trajectory that shares the property of being a solution of the non-autonomous Hamiltonian equations and of connecting instantaneous (i.e., at fixed time) hyperbolic points (so called $X$-points), see, e.g., Refs. 30 and 45. It has the property that all neighbouring field lines approach such a trajectory exponentially either forward or backward in time. Stable and unstable manifolds are invariant surfaces defined as the set of trajectories that converge towards the DHT forward or backward in time, respectively. The intersection of these manifolds with a $t=$ const surface defines one dimensional curves. A sketch of these curves and of the lobes produced by the convoluted shape of the stable and the unstable manifolds in the proximity of two DHTs is shown in Fig. 1.

It can be shown, along the lines of Refs. 48, 55, and 56 that, the boundary $P_{2} O P_{1}$, taken along the unstable manifold of $P_{2}$ and the stable manifold of $P_{1}$ after selecting the intersection point $O$, partitions the phase space represented in Fig. 1 into macro-regions where trajectories have a qualitatively different behaviour. How fast mixing can occur

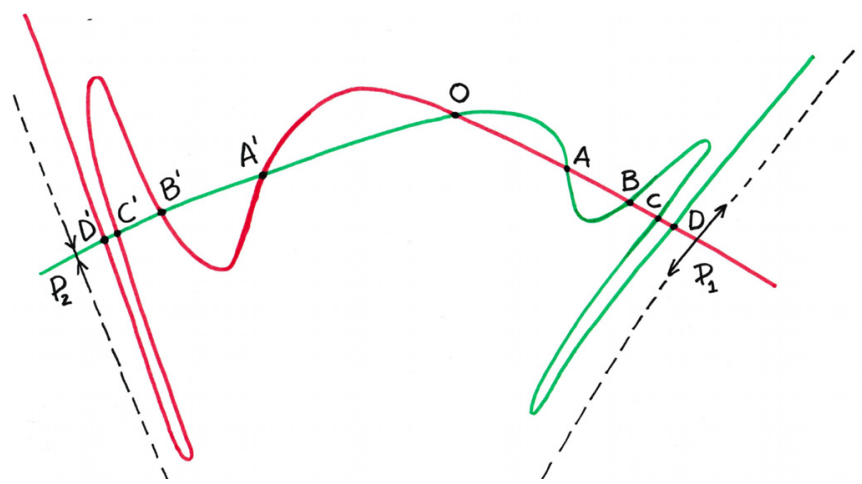

FIG. 1. Sketch of the lobes produced by the convoluted shape of the stable and the unstable manifolds in the proximity of two DHTs. 
through this boundary depends on the shape of the intersecting invariant manifolds.

\section{TRANSPORT PHENOMENA IN NON-AUTONOMOUS, NON PERIODIC, DYNAMICAL SYSTEMS WITH ONE DEGREE OF FREEDOM}

In Sec. III, we have briefly recalled how it is possible to characterize transport processes in periodic systems using the geometry of stable and unstable manifolds. These structures can be calculated knowing the trajectories and the velocity field of the dynamical system for the finite time interval given by the system periodicity.

This is not the case of non-periodic-systems. In fact, in the most general case, knowledge of the velocity field is required for an infinite time interval in order to define invariant manifolds and lobes. For this reason, a different technique, based on the definition and identification of the so-called Lagrangian Coherent Structures (LCSs), e.g., see Refs. 2, 3, and 19, has been developed in order to study transport processes for the most general non-periodic flows.

As in the periodic case, the aim is to identify domains in phase space with a different dynamical behaviour and, eventually, transport features. The LCSs are the boundaries of these regions of coherence. In contrast to the invariant manifolds, LCSs are defined over a finite amount of time, $\tau$, related to the characteristic time of coherence of the motion. As $\tau$ increases, LCS converges to the invariant manifolds mentioned above, see Ref. 2. We note in passing that, although not directly relevant to our problem, the concept of LCS does not require the system to be Hamiltonian.

Following Refs. 22 and 24, in this work, we consider only hyperbolic LCSs which organize the Hamiltonian flow by attracting or repelling volume elements of phase space over the finite time span $\tau$. For the sake of simplicity, we will refer to these structures simply as LCS. The rigorous definition of these structures has been subject to debate: see, e.g., Refs. 3, 19, and 24. The first way of finding LCS was based on constructing the field of the finite time Lyapunov exponents (FTLE), see Refs. 19, 22, and 23. Since at a given phase space position the largest positive FTLE measures the exponential separation between two neighbouring initial conditions after a given interval of time, within this formulation LCSs have been defined as second-derivative ridges of the FTLE-field. Several counter examples to this heuristic definition have been found by Haller who introduced the definition of hyperbolic LCS, see Ref. 3, as the most repulsive or attractive material lines, where material lines are defined as lines of initial conditions advected by the Hamiltonian flow.

\section{LAGRANGIAN COHERENT STRUCTURES (LCSS)}

In this section, we briefly recall the definition of LCSs (see Refs. 3 and 24). As mentioned before, we consider a dynamical system in 2D phase space $\boldsymbol{x}=(x, y)$

$$
\frac{d x}{d t}=v_{x}(t, x, y), \quad \frac{d y}{d t}=v_{y}(t, x, y)
$$

with continuous differentiable flow map

$$
\phi_{t_{0}}^{t}\left(x_{0}\right)=\boldsymbol{x}\left(t, t_{0}, x_{0}\right)
$$

Two neighbouring points $\boldsymbol{x}_{0}$ and $\boldsymbol{x}_{0}+\boldsymbol{\delta} x_{0}$ evolve into the points $\boldsymbol{x}$ and $\boldsymbol{x}+\boldsymbol{\delta} x$ under the linearized map

$$
\boldsymbol{\delta} x=\nabla \phi_{t_{0}}^{t} \boldsymbol{\delta} x_{0}
$$

Let us consider a curve $\gamma_{0}=\left\{\boldsymbol{x}_{0}=r(s)\right\}$ and at each point $\boldsymbol{x}_{0} \in \gamma_{0}$ let us define the unit tangent vector $\boldsymbol{e}_{0}$ and the normal vector $\boldsymbol{n}_{0}$. In the time interval $\left[t_{0}, t\right]$, the dynamics of the system advects the material line $\gamma_{0}$ into $\gamma_{t}$ and $x_{0} \in \gamma_{0}$ into $\boldsymbol{x}_{t} \in \gamma_{t}$. The linearized dynamics maps the tangent vector $\boldsymbol{e}_{0}$ into $\boldsymbol{e}_{t}$ which is tangent to $\gamma_{t}$ and is given by

$$
\boldsymbol{e}_{t}=\frac{\nabla \phi_{t_{0}}^{t}\left(\boldsymbol{x}_{0}\right) \boldsymbol{e}_{0}}{\left[\boldsymbol{e}_{0} \boldsymbol{C}_{t_{0}}^{t}\left(\boldsymbol{x}_{0}\right) \boldsymbol{e}_{0}\right]^{1 / 2}},
$$

where $\boldsymbol{C}_{t_{0}}^{t}\left(\boldsymbol{x}_{0}\right) \equiv\left(\boldsymbol{\nabla} \phi_{t_{0}}^{t}\right)^{T} \boldsymbol{\nabla} \phi_{t_{0}}^{t}$ is the Cauchy-Green strain tensor and ${ }^{T}$ stands for transposed. This symmetric tensor describes the deformation of an arbitrary small circle of initial conditions, centered in $\boldsymbol{x}_{0}$ caused by the flow in a time interval $\left[t_{0}, t\right]$. Taking, for example, a circle centered in $\boldsymbol{x}_{0}$ with radius $\left\|\delta \boldsymbol{x}_{0}\right\|$, after the time interval $\left[t_{0}, t\right]$ it will be deformed into an ellipse with major axis in the direction of $\boldsymbol{\xi}_{\text {max }}$ and minor axis in the direction of $\boldsymbol{\xi}_{\text {min }}$, where $\boldsymbol{\xi}_{\text {max }}$ and $\boldsymbol{\xi}_{\text {min }}$ are the two eigenvectors of $\boldsymbol{C}_{t_{0}}^{t}\left(\boldsymbol{x}_{0}\right)$. The corresponding real and positive eigenvalues are $\lambda_{\text {max }}$ and $\lambda_{\text {min }}$. The curves with tangent vector along $\xi_{\text {min }}$ and, respectively, $\xi_{\text {max }}$ are called strain lines of the Cauchy-Green tensor. In general, the mapping does not preserve the angle between vectors and therefore usually $\boldsymbol{n}_{t}$ differs from $\boldsymbol{\nabla} \phi_{t_{0}}^{t} \boldsymbol{n}_{0}$.

Using the orthogonality condition $\boldsymbol{n}_{0} \cdot \boldsymbol{e}_{0}=\boldsymbol{n}_{0} \boldsymbol{\nabla} \phi_{t}^{t_{0}} \nabla \phi_{t_{0}}^{t} \boldsymbol{e}_{0}$ $=0$ and inserting Eq. (6), we obtain the expression for $\boldsymbol{n}_{t}$ which is given by

$$
\boldsymbol{n}_{t}=\frac{\left(\boldsymbol{\nabla} \phi_{t}^{t_{0}}\right)^{T} \boldsymbol{n}_{0}}{\left[\boldsymbol{n}_{0} \boldsymbol{C}^{-1}\left(\boldsymbol{x}_{0}\right) \boldsymbol{n}_{0}\right]^{1 / 2}},
$$

where $\boldsymbol{C}^{-1}\left(\boldsymbol{x}_{0}\right)=\boldsymbol{C}_{t}^{t_{0}}\left(\boldsymbol{x}_{0}\right)$ and the time interval marks have been suppressed as will be the case in the following formulae when not explicitly needed.

We define the repulsion ratio $\rho_{t_{0}}^{t}\left(\boldsymbol{x}_{0}, \boldsymbol{n}_{0}\right)$ as the ratio at which material points, in other words points advected by the flow, initially taken near the point $\boldsymbol{x}_{0} \in \gamma_{0}$, increase their distance from the curve in the time interval $\left[t_{o}, t\right]$

$$
\rho_{t_{0}}^{t}\left(\boldsymbol{x}_{0}, \boldsymbol{n}_{0}\right)=\boldsymbol{n}_{t} \nabla \phi_{t_{0}}^{t}\left(\boldsymbol{x}_{0}\right) \boldsymbol{n}_{0}
$$

Using the previous definitions, $\rho_{t_{0}}^{t}\left(\boldsymbol{x}_{0}, n_{0}\right)$ can be expressed either in terms of $n_{0}$ or of $n_{t}$ as

$$
\rho_{t_{0}}^{t}\left(\boldsymbol{x}_{0}, n_{0}\right)=\left[\boldsymbol{n}_{0} \boldsymbol{C}^{-1}\left(\boldsymbol{x}_{0}\right) \boldsymbol{n}_{0}\right]^{-1 / 2}=\left[\boldsymbol{n}_{t} \boldsymbol{C}\left(\boldsymbol{x}_{0}\right) \boldsymbol{n}_{t}\right]^{1 / 2} .
$$

Similarly, the contraction rate $L_{t_{0}}^{t}\left(\boldsymbol{x}_{0}\right)$ is proportional to the growth in time of the vector tangent to the material line

$$
L\left(\boldsymbol{x}_{0}, \boldsymbol{e}_{0}\right)=\left[\boldsymbol{e}_{0} \boldsymbol{C}\left(\boldsymbol{x}_{0}\right) \boldsymbol{e}_{0}\right]^{1 / 2} .
$$




\section{A. LCS as maximal repulsion-attraction material lines}

Here, we adopt the definition of a Hyperbolic LCS as given in Ref. 3. An LCS over a finite time interval $\left[t_{0}, t_{0}+T\right]$ is defined as a material line along which the repulsion rate is pointwise maximal. This leads, as shown in Refs. 3 and 24, to the following definitions.

A material line satisfying the following conditions at each point:

$$
\begin{array}{ll}
\text { a) } & \lambda_{\text {min }}<\lambda_{\text {max }}, \quad \lambda_{\text {max }}>1, \\
\text { b) } & \boldsymbol{e}_{0}=\boldsymbol{\xi}_{\text {min }},
\end{array}
$$

the tangent vector is along the eigenvector associated with the smallest eigenvalue

$$
\text { c) } \quad \boldsymbol{\xi}_{\max } \cdot \nabla \lambda_{\max }=0
$$

the gradient of the largest eigenvalue is along the curve, is called a repulsive Weak Lagrangian Coherent Structure (WLCS).

A WLCS which satisfies at each point the additional condition

$$
\xi_{\text {max }} \cdot \nabla^{2} \lambda_{\text {max }} \cdot \boldsymbol{\xi}_{\text {max }}<0
$$

is called a repulsive Lagrangian coherent structure. Attractive LCSs are defined as repulsive LCS of the backward time dynamics.

Finally, we note that in the case of a Hamiltonian nonautonomous system with one degree of freedom phase space conservation implies

$$
\lambda_{\text {min }} \lambda_{\text {max }}=1
$$

We note that a major difference with respect to the definition of LCS based on the second derivative ridges in Ref. 19 is that the definition of LCS as maximal repulsionattraction material lines involves the eigenvectors and eigenvalues of the Cauchy-Green strain tensor $\boldsymbol{C}$ while the definition in terms of the second derivative ridge is governed by the eigenvectors and eigenvalues of the Hessian $\boldsymbol{\Sigma}$ of FTLE field $\sigma$ defined as

$$
\sigma\left(x_{0}, t_{0}, t\right)=\frac{1}{2\left|t-t_{0}\right|} \ln \lambda_{\max }\left(\boldsymbol{x}_{0}, t_{0}, t\right) .
$$

In Ref. 24, an elementary example is discussed where a WLCS is explicitly shown not to be a second derivative ridge.

\section{RECONNECTING MAGNETIC CONFIGURATION}

As mentioned in the Introduction, the aim of this and its accompanying paper is to obtain information about particle transport due to the onset of magnetic reconnection from the behaviour of the magnetic field lines. The reconnection setting that we adopt is the same as that used in Ref. 22, which is based on a numerical simulation where reconnection is made possible by the effect of electron inertia, Ref. 57. The reconnecting magnetic field has only components in the $x-y$ plane but depends on all the three spatial coordinates. In this numerical simulation, the magnetic field evolution starts from a static equilibrium, expressed in terms of a magnetic flux function $\psi_{e q}$ as

$$
\mathbf{B}_{e q}=B_{0} \mathbf{e}_{z}+\nabla \psi_{e q}(x) \times \mathbf{e}_{z},
$$

with $\psi_{e q}=0.19 \cos (x)$. Periodicity is assumed in all three directions and the configuration is restricted to the domain $\left[-L_{x}, L_{x}\right] \times\left[-L_{y}, L_{y}\right] \times\left[-L_{z}, L_{z}\right] \quad$ with $L_{x}=\pi, L_{y}=2 \pi, L_{z}$ $=16 \pi$.

In Ref. 57, a "double helicity" perturbation (i.e., in the considered planar geometry, a perturbation made of two components with different phase planes) is initially imposed

$$
\begin{aligned}
\hat{\psi}(x, y, z, t)= & \hat{\psi}_{1}(x, t) \cos \left(k_{1 y} y+k_{1 z} z\right) \\
& +\hat{\psi}_{2}(x, t) \cos \left(k_{2 y} y+k_{2 z} z\right),
\end{aligned}
$$

where $k_{1 y}=k_{2 y}=2 \pi / L_{y}$ and $k_{1 z}=0$ while $k_{2 z}=2 \pi / L_{z}$. The eigenfunctions of the initial perturbations, $\hat{\psi}_{1}(x, 0)$ and $\hat{\psi}_{2}(x, 0)$, are localized functions on the resonant surfaces and the initial amplitude of $\hat{\psi}_{1}$ was chosen to be of order $10^{-4}$ and ten times bigger than that of $\hat{\psi}_{2}$. The resonant surfaces $x=x_{i}$ are defined by the condition $\mathbf{B}_{e q} \cdot \mathbf{k}_{1,2}=0$ and, disregarding the mirror-doubling of the configuration caused by the assumed periodicity along $x$, are located at $x_{1}=0$ and $x_{2}=0.71$, respectively.

As is well known, perturbations with different "helicities" are required in order to make the Hamiltonian system described in Sec. II non-integrable, i.e., to generate a chaotic magnetic configuration. In the following, we will denote by $\psi(x, y, z, t)=\psi_{e q}(x)+\hat{\psi}(x, y, z, t)$ the complete magnetic flux function that includes the equilibrium and the physical time evolving perturbations. At each fixed physical time $t$, it plays the role of the Hamiltonian for the magnetic field lines while the space coordinates $x$ and $y$ that of canonical variables with $x$ the momentum and $y$ the position. The field line Eq. (2) becomes

$$
\frac{d x}{d z}=-\frac{\partial \psi}{\partial y}, \quad \frac{d y}{d z}=\frac{\partial \psi}{\partial x} .
$$

In the linear phase, when the two components of the perturbation evolve independently without interacting with each other, two chains of magnetic islands are formed around their own resonant surfaces.

During their evolution, the magnetic islands expand and start to interact making the linear approximation invalid. The dynamics of the magnetic configuration becomes rapidly nonlinear and higher order modes are spontaneously generated. The most relevant of these nonlinear modes turn out to have the same helicities of the two components of the imposed perturbation. At this stage, the magnetic field topology exhibits regions where field lines are stochastic, and these regions tend to spread as the reconnection process evolves. A detailed description of the chaos inception and spread all over the domain of the configuration can be found in Refs. 30 and 57.

In the numerical investigation in Paper II, we will focus on two different normalized physical times, $t=415$ and 
$t=425$ Ref. 30 (normalized respect to the Alfvén time defined in terms of $\psi_{e q}$ ), in which the chaos, initially developed only on a local scale (at $t=415$ ), starts to spread on a global scale (at $t=425$ ). At the normalized physical times $t=415$ and $t=425$, the amplitude of the perturbed magnetic field is of the order of the shear equilibrium field given by the second term in Eq. (17) and particles following the perturbed magnetic field lines experiment large excursions along the $x$ direction. In this advanced phase of the reconnection process with almost overlapping island chains, as indicated by the Poincarè map in Fig. 2 , the $x$-component of the particle velocity, as obtained by projecting on the $x-y$ plane the particle velocity along field lines, is much larger than the particle drift velocities that are proportional to the ratio between the particle gyroradius and the scale length of the magnetic field inhomogeneity. This scale separation is even more evident in the case of electrons and in general of particles with velocities along field lines larger than in the perpendicular directions, i.e., of the particles whose transport properties are most affected by magnetic fluctuations.

\section{A. Time periodic dynamical system}

First, we consider the dynamical system that is obtained by taking a snapshot at a given physical time $t=\bar{t}$ of the reconnecting magnetic configuration following the procedure introduced in Sec. II, where the flux function $\psi(x, y, z, t=\bar{t})$ is the Hamiltonian and $z$ is the magnetic Hamiltonian time. Since the configuration is periodic in $z$ with periodicity $32 \pi$, we adopt the Poincaré map technique and compare it with the LCS approach.

The magnetic configuration in Sec. VI is symmetric under the space-time reflection symmetry $y \rightarrow-y, z \rightarrow-z$ since $\psi(x, y, z, t=\bar{t})=\psi(x,-y,-z, t=\bar{t})$. This property can be exploited when computing attractive LCSs as they can be seen as repulsive LCSs with respect to the inverted "time" $-z$. Because of the above reflection symmetry, this time inversion is equivalent to setting $y \rightarrow-y$, i.e., the attractive LCSs are mirror images of the repulsive LCSs with respect to $y=0$.

\section{B. Time nonperiodic dynamical system}

A rudimentary way to take into account the fact that the magnetic configuration changes during the particle transit time is to adopt a model where the particle gyromotion and drifts are

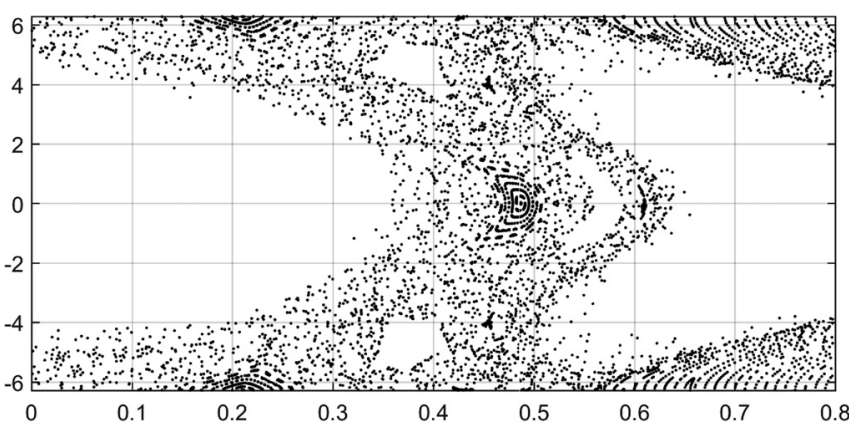

FIG. 2. Poincaré map at $z=0$ and $t=425$ showing the chaoticity region between the two island chains. neglected and the particles dynamics is only included through their streaming velocity $V$ along the guide field $B_{0}$, i.e., along $z$. Furthermore, $V$ is assumed to stay constant. As mentioned in the Introduction, this model oversimplifies the description of the particle transport caused to the onset of magnetic reconnection. However, it allows us to describe LCSs in a time nonperiodic dynamical system and, most importantly, to include kinetic-type effects by defining LCSs that depend explicitly on the different particle velocities.

With this in mind, we introduce a family of nonautonomous dynamical systems in the extended phase space $x, y, z$, with $z$ playing again the role of time and each system being characterized by a different velocity $V$, by introducing the Hamiltonian

$$
\psi_{\mathcal{V}}(x, y, z) \equiv \psi\left(x, y, z, t=\left(z-z_{o}\right) / V\right) .
$$

Here $t$ is taken to be positive and, in fact, it is defined at $t-t_{1}$ where $t_{1}$ is the physical time at which we start our investigation of the particle trajectories while, for convenience, we set the starting magnetic Hamiltonian time $z_{o}=0$. In the following, we will restrict the range over which the physical time $t$ varies to the interval $t_{1}<t<t_{2}$. As mentioned above, at $t_{1}=415$, i.e., in the early stage of the nonlinear reconnection process, the regions of chaoticity of the magnetic field lines are still separated. At the later stage $t_{2}=425$ chaotic regions merge and the system experiences a transition from local to global chaoticity.

\section{Positive and negative velocities}

For positive velocities, the physical time $t=z / V$ that appears in the Hamiltonian $\psi\left(x, y, z, t=\left(z-z_{o}\right) / V\right)$ increases as $z$ increases and the new Hamilton equations read

$$
\frac{d x}{d z}=-\frac{\partial \psi_{\mathcal{V}}}{\partial y}, \quad \frac{d y}{d z}=\frac{\partial \psi_{\mathcal{V}}}{\partial x} .
$$

On the contrary for negative velocities, $z$ decreases as $t$ increases. Thus for negative velocities, it is convenient to refer to the variables $\zeta=-z$ and $\eta=-y$ and write

$$
\begin{aligned}
\psi_{-|\mathcal{V}|}(x, y, z) & \equiv \psi(x, y, z, t=-z /|V|) \\
& =\psi(x, \eta, \zeta, t=\zeta /|V|),
\end{aligned}
$$

where we have used the symmetry at fixed time $t$, so that $\psi_{-|\mathcal{V}|}(x, y, z) \equiv \psi_{|\mathcal{V}|}(x, \eta, \zeta)$.

Then for negative velocities the Hamilton equations (22) can be rewritten in the form

$$
\frac{d x}{d \zeta}=-\frac{\partial \psi_{|\mathcal{V}|}}{\partial \eta}, \quad \frac{d \eta}{d \zeta}=\frac{\partial \psi_{|\mathcal{V}|}}{\partial x} .
$$

which shows that the trajectories for positive and negative velocities differ since they are determined by the same Hamiltonian but involve different spatial and time domains.

\section{Repulsive and attractive velocity dependent LCS}

As shown above, the time periodic Hamiltonian attractive LCSs are simply mirror images of the repulsive LCSs 
with respect to $y=0$. In the case of the time nonperiodic Hamiltonian, this result is no longer valid.

Here, for the sake of clarity we consider only positive velocities $V$. For attractive LCS, we start from $t=t_{2}$ and $z_{\text {fin }}=z+\left(t_{2}-t\right) V$ and find it convenient to define a new Hamiltonian function (with a reversed sign because of the inversion of the time variable $z$ )

$$
\bar{\psi}\left(x, y, z_{f i n}-z, t_{2}-t\right)=-\psi(x, y, z, t),
$$

and the variable $\bar{\zeta}=z_{f i n}-z$, (shifted with respect to the variable $\zeta$ defined in Sec. VIB 1). At fixed physical time $t$ we have

$$
\frac{d x}{d z}=-\frac{\partial \psi}{\partial y} \rightarrow \frac{d x}{d \bar{\zeta}}=-\frac{\partial \bar{\psi}}{\partial y}, \quad \frac{d y}{d z}=\frac{\partial \psi}{\partial x} \rightarrow \frac{d y}{d \bar{\zeta}}=\frac{\partial \bar{\psi}}{\partial x} .
$$

Proceeding as for Eq. (20) we write

$$
\bar{\psi}_{\mathcal{V}}(x, y, \bar{\zeta})=\bar{\psi}(x, y, \bar{\zeta}, \bar{\zeta} / V)
$$

and obtain

$$
\frac{d x}{d \bar{\zeta}}=-\frac{\partial \bar{\psi}_{\mathcal{V}}}{\partial y}, \quad \frac{d y}{d \bar{\zeta}}=\frac{\partial \bar{\psi}_{\mathcal{V}}}{\partial x},
$$

which shows that the equations for the attractive LCS are the same in form as those for the repulsive LCS but with a different "time" variable, $\bar{\zeta}$, and a different Hamiltonian, $\bar{\psi}_{V}$.

\section{CONCLUSIONS}

In the first (Paper I) of a pair of linked papers, we have presented the theoretical framework that will be used in Paper II for the numerical investigation of the Lagrangian Coherent Structures (LCSs) seen by plasma particles restricted to move along the magnetic field lines of a magnetic configuration that evolves in physical time because of magnetic reconnection. Our aim is to identify macro-regions distinguished by a qualitatively different behaviour of the particle motion. The main limitation of this simplified model arises from this restriction on the particle motion that, however, it plays a very convenient role as it allows us to extend the scope of the well-known relationship between field line equations and the dynamics of a non-autonomous dynamical system with one degree of freedom.

Clearly this restriction can be overcome by inserting into Eq. (3) a more realistic expression for the particle motion, as obtained, e.g., in the gyrocenter approximation (see Refs. 58 and 59) once the magnetic and electric field configurations and their time evolution are known. In order to maintain a description that is two-dimensional in space plus time, as in the simplified treatment described above, the particle trajectories need to be expressed with respect to a coordinate along field lines that plays the role of time. This reparametrization may require that the particles be first divided into different classes depending on their orbit topology as is the case, for a toroidal plasma, of passing and trapped particles (see Ref. 60). Finally, we stress that a wide range of application of the LCS approach to different plasma configurations is easy to envision. For example, LCSs can offer a new approach for the study of anomalous particle transport in space or astrophysical plasmas where this technique may complement investigations performed with different tools, see, e.g., Ref. 61 for heliospheric plasmas. Conversely, LCS may be looked for in a kinetic plasma description in order to identify transport structures in particle phase space. An investigation of this type was performed in the case of a beam plasma instability in Ref. 9 in terms of a one dimensional Vlasov-Poisson system.

\section{ACKNOWLEDGMENTS}

G.D.G. and D.G. thank Dario Borgogno for fruitful discussions.

${ }^{1}$ G. Haller and G. Yuan, Phys. D: Nonlinear Phenom. 147, 352 (2000).

${ }^{2}$ G. Haller, Annu. Rev. Fluid Mech. 47, 137 (2015).

${ }^{3}$ G. Haller, Phys. D: Nonlinear Phenom. 240, 574 (2011).

${ }^{4}$ C. Coulliette, F. Lekien, J. D. Paduan, G. Haller, and J. E. Marsden, Environ. Sci. Technol. 41, 6562 (2007).

${ }^{5}$ S. C. Shadden and C. A. Taylor, Ann. Biomed. Eng. 36, 1152 (2008).

${ }^{6}$ F. Huhn, A. von Kameke, V. Pérez-Muñuzuri, M. Olascoaga, and F. Beron-Vera, Geophys. Res. Lett. 39, L06602, https://doi.org/10.1029/ 2012GL051246 (2012).

${ }^{7}$ J. Peng and J. Dabiri, J. Fluid Mech. 623, 75 (2009).

${ }^{8}$ W. Tang, M. Mathur, G. Haller, D. C. Hahn, and F. H. Ruggiero, J. Atmos. Sci. 67, 2307 (2010).

${ }^{9}$ N. Carlevaro, M. V. Falessi, G. Montani, and F. Zonca, J. Plasma Phys. 81, 495810515 (2015).

${ }^{10}$ A. C.-L. Chian, E. L. Rempel, G. Aulanier, B. Schmieder, S. C. Shadden, B. T. Welsch, and A. R. Yeates, Astrophys. J. 786, 51 (2014).

${ }^{11}$ E. L. Rempel, A. C.-L. Chian, A. Brandenburg, P. R. Muñoz, and S. C. Shadden, J. Fluid Mech. 729, 309 (2013).

${ }^{12}$ J. Szezech, Jr., I. Caldas, S. Lopes, P. J. Morrison, and R. L. Viana, Phys. Rev. E 86, 036206 (2012).

${ }^{13}$ J. Szezech, Jr., A. Schelin, I. Caldas, S. Lopes, P. J. Morrison, and R. L. Viana, Phys. Lett. A 377, 452 (2013).

${ }^{14}$ K. Padberg, T. Hauff, F. Jenko, and O. Junge, New J. Phys. 9, 400 (2007).

${ }^{15}$ L. C. de Oliveira, C. G. L. Martins, M. Roberto, I. L. Caldas, and E. R. de Carvalho, Phys. A 391, 6611 (2012).

${ }^{16}$ M. Budisica and J. L. Thiffeault, Chaos 25, 087407 (2015).

${ }^{17}$ J. L. Thiffeault, Chaos 20, 017516 (2010).

${ }^{18}$ M. R. Allshouse and J. L. Thiffeault, Phys. D: Nonlinear Phenom. 241, 95 (2012).

${ }^{19}$ S. C. Shadden, F. Lekien, and J. E. Marsden, Phys. D: Nonlinear Phenom. 212, 271 (2005).

${ }^{20}$ M. Farazmand and G. Haller, Chaos 22, 013128 (2012).

${ }^{21}$ K. Onu, F. Huhn, and G. Haller, J. Comput. Sci. 7, 26 (2015).

${ }^{22}$ D. Borgogno, D. Grasso, F. Pegoraro, and T. Schep, Phys. Plasmas 18, 102307 (2011).

${ }^{23}$ G. Rubino, D. Borgogno, M. Veranda, D. Bonfiglio, S. Cappello, and D. Grasso, Plasma Phys. Controlled Fusion 57, 085004 (2015).

${ }^{24}$ M. Falessi, F. Pegoraro, and T. Schep, J. Plasma Phys. 81, 495810505 (2015).

${ }^{25}$ M. Veranda, D. Bonfiglio, S. Cappello, D. F. Escande, F. Auriemma, D. Borgogno, L. Chacón, A. Fassina, P. Franz, M. Gobbin et al., Nucl. Fusion 57, 116029 (2017).

${ }^{26}$ D. Borgogno, P. Anna, and D. Grasso, Phys. Plasmas 24, 122303 (2017).

${ }^{27}$ A. Rechester and M. Rosenbluth, Phys. Rev. Lett. 40, 38 (1978).

${ }^{28}$ S. Abdullaev, Magnetic Stochasticity in Magnetically Confined Fusion Plasmas, Springer Series on Atomic, Optical, and Plasma Physics, Vol. 78 (Springer, 2014).

${ }^{29}$ H. Wobig and D. Pfirsch, Plasma Phys. Controlled Fusion 43, 695 (2001).

${ }^{30}$ D. Borgogno, D. Grasso, F. Pegoraro, and T. Schep, Phys. Plasmas 15, 102308 (2008)

${ }^{31}$ J. R. Cary and R. G. Littlejohn, Ann. Phys. 151, 1 (1983).

${ }^{32}$ M. D. Kruskal, Project Matterhorn Report No. NYO-998, PM-S-5, Princeton University Forrestal Research Center, 1952. See National Technical Information Service Document No. PB200-100659 (21 pages of 
"Some properties of Rotational Transforms"). Copies may be ordered from the National Technical Information Service, Springfield, VA 22161.

${ }^{33}$ D. Kerst, J. Nucl. Energy Part C 4, 253 (1962).

${ }^{34}$ I. M. Gel'fand, M. I. Graev, N. M. Zueva, A. I. Morozov, and L. S. Solov'ev, "Magnetic Surfaces of Triple-Layer Helical Magnetic Field Disturbed by Crimped Field," Sov. Phys.-Tech. Phys. 31 (1961).

${ }^{35}$ A. Morozov and L. Solov'ev, in Reviews of Plasma Physics, edited by M. A. Leontovich (Consultants Bureau, New York, 1966) Vol. 2, pp. 201-297.

${ }^{36}$ A. H. Boozer, Phys. Fluids 24, 1999 (1981).

${ }^{37}$ M. Rosenbluth, R. Sagdeev, J. Taylor, and G. Zaslavski, Nucl. Fusion 6, 297 (1966).

${ }^{38}$ K. Elsasser, Plasma Phys. Controlled Fusion 28, 1743 (1986).

${ }^{39}$ P. Morrison, Phys. Plasmas 7, 2279 (2000).

${ }^{40}$ A. H. Boozer, Rev. Mod. Phys. 76, 1071 (2005).

${ }^{41}$ T. E. Evans, R. A. Moyer, and P. Monat, Phys. Plasmas 9, 4957 (2002).

${ }^{42}$ I. L. Caldas, B. F. Bartoloni, D. Ciro, G. Roberson, A. B. Schelin, T. Kroetz, M. Roberto, R. L. Viana, K. C. Iarosz, A. M. Batista, and P. J. Morrison, "Symplectic Maps for Diverted Plasmas," IEEE Trans. Plasma Sci. (published online 2018).

${ }^{43}$ S. Abdullaev, Nucl. Fusion 54, 064004 (2014).

${ }^{44}$ V. I. Arnold, V. V. Kozlov, and A. I. Neishtadt, Mathematical Aspects of Classical and Celestial Mechanics (Springer Science \& Business Media, Berlin, 2007), Vol. 3.

${ }^{45}$ S. Wiggins, Phys. Today. 45(7), 68 (1992).

${ }^{46}$ A. Vulpiani, Chaos: From Simple Models to Complex Systems (World Scientific, Singapore, 2010), Vol. 17.
${ }^{47}$ S. Abdullaev, Construction of Mappings for Hamiltonian Systems and Their Applications (Springer, 2006), Vol. 691.

${ }^{48}$ R. S. Mackay, J. Meiss, and I. Percival, Phys. D: Nonlinear Phenom. 27, 1 (1987).

${ }^{49}$ J. Meiss, Chaos 25, 097602 (2015).

${ }^{50} \mathrm{~J}$. M. Ottino, The Kinematics of Mixing: Stretching, Chaos, and Transport (Cambridge University Press, Cambridge, 1989), Vol. 3.

${ }^{51}$ S. Wiggins, Chaotic Transport in Dynamical Systems (Springer Science \& Business Media, New York, 2013), Vol. 2.

${ }^{52}$ V. Rom-Kedar and S. Wiggins, Archive Rational Mech. Anal. 109, 239 (1990).

${ }^{53}$ V. Rom-Kedar, A. Leonard, and S. Wiggins, J. Fluid Mech. 214, 347 (1990).

${ }^{54}$ N. Malhotra and S. Wiggins, J. Nonlinear Sci. 8, 401 (1998).

${ }^{55}$ Q. Chen, Phys. Lett. A 123, 444 (1987).

${ }^{56} \mathrm{~F}$. Raynal and S. Wiggins, Phys. D: Nonlinear Phenom. 223, 7 (2006).

${ }^{57}$ D. Borgogno, D. Grasso, F. Porcelli, F. Califano, F. Pegoraro, and D. Farina, Phys. Plasmas 12, 032309 (2005).

${ }^{58}$ E. Frieman and L. Chen, Phys. Fluids 25, 502 (1982).

${ }^{59}$ A. J. Brizard and T. S. Hahm, Rev. Mod. Phys. 79, 421 (2007).

${ }^{60}$ F. Hinton and R. Hazeltine, Rev. Mod. Phys. 48, 239 (1976).

${ }^{61}$ G. Zimbardo, S. Perri, P. Pommois, and P. Veltri, Adv. Space Res. 49, 1633 (2012).

${ }^{62}$ G. Di Giannatale, M. V. Falessi, D. Grasso, F. Pegoraro, and T. J. Schep, Phys. Plasmas 25, 052307 (2018). 